\title{
Eine Geldverwaltung gibt zu reden
}

\author{
Heini Bader (Zürich)
}

Zusammenfassung: Anhand von Fallvignetten aus einer langjährigen Geldverwaltung wird dargestellt, wie im traditionellen Feld von Sozialarbeit Elemente psychoanalytischen Verstehens Anwendung finden können. Dabei kommen die verschiedenen triangulierenden Aspekte der Geldverwaltung zur Sprache.

Schlüsselwörter: Psychoanalytische Sozialarbeit, Geldverwaltung, Setting, Sucht, Triangulierung

Das Thema Geld erscheint in der psychoanalytischen Literatur in verschiedenen Zusammenhängen. Die Anwendung von psychoanalytischen Konzepten auf Schuldenprobleme und Geldverwaltungen wird meines Wissens hingegen nirgends behandelt.

In programmatischer Weise formuliert Michael Günter wesentliche Elemente zur Arbeitsweise von psychoanalytischer Sozialarbeit: « ... psychoanalytische Sozialarbeit (hat) sich in ihrem Selbstverständnis immer mit dem Verhältnis von gemeiner Lebenswirklichkeit und inneren Fantasien zu beschäftigen und spezifische Ansätze für eine bessere Verknüpfung dieser beiden Wirklichkeitsbereiche für den jeweiligen Patienten und dessen Probleme zu entwickeln. Als Sozialarbeit setzt sie an der Lebenswelt der Patienten an, wohl wissend, dass diese nicht nur an den äusseren Verhältnissen und der mangelnden eigenen Stärke und Durchsetzungsfähigkeit scheitern, sondern auch daran, dass unsere Patienten gezwungen sind, ihre inneren Konflikte im Sozialen zu organisieren. Die psychoanalytische Perspektive ermöglicht das Verständnis der inneren Brüche nicht nur in der Übertragungsbeziehung der therapeutischen Einzelstunde, sondern auch ein Verstehen der sich in den Alltagsbeziehungen breit machenden komplexen Übertragungs-Gegenübertragungskonstellationen. Letztere bestimmten das Interaktionsgeschehen bei unseren Patienten so weitgehend, dass rein pädagogische Ansätze häufig rasch zum Scheitern verurteilt sind, weil sie der Verwicklung in maligne Übertragungs-Gegenübertragungsdynamiken kaum angemessene Reflexions- und damit Distanzierungsmöglichkeiten entgegenzusetzen haben. Erst die Reflexion darauf, welche psychischen Konflikte und Inkompatibilitäten im seelischen Leben der Patienten sie zu derartigen Handlungen treiben, erlauben 
es aus der Welt der Sachen, in der das andere rücken muss, wenn das eine Platz nimmt, in eine Welt der Gedanken zurückzukehren, in der verschiedene Optionen nebeneinander bestehen bleiben können.

So gesehen hat Psychoanalytische Sozialarbeit mit der Rückübersetzung der Ereignisse in der realen Welt in Formen des Denkens zu tun, in Formen der Entwicklung von Sinn gebenden Geschichten» (Günter 2005: 87).

Anhand von Schilderungen einzelner Situationen aus einer mehr als sechs Jahre dauernden Geldverwaltung will ich Elemente dieser Arbeitsweise darzustellen versuchen.

Doch zunächst möchte ich darlegen, wie wir das Setting von Geldverwaltungen verstehen.

Geldverwaltungen werden als freiwillige oder erzwungene Massnahmen traditionellerweise im Rahmen von vormundschaftlichen Betreuungsverhältnissen durchgeführt. Sie stellen einen tiefen Eingriff in die Selbstständigkeit der KlientInnen dar und konstituieren ein Machtverhältnis in der Realität, welches für die sich entwickelnde Übertragungsbeziehung von allergrösster Bedeutung ist. Es ist deshalb angezeigt, ein Setting zu konstruieren, das diesen Verhältnissen Rechnung trägt.

Dabei ist das wichtigste Element die Triangulierung: Individuell abgestimmte Regeln gewährleisten, dass die Einnahmen der KlientInnen (Lohn, Rente, Sozialgeld) mittels Abtretungserklärungen über das Treuhandkonto des Vereins (vpsz) laufen. Ebenso erfolgen alle Zahlungen über dieses Konto. Die Buchhaltungsunterlagen werden regelmässig durch aussenstehende RevisorInnen einer strengen Kontrolle unterzogen, womit eine Instanz geschaffen ist, die Regeln setzt und überprüft, welche für den psychoanalytischen Sozialarbeiter wie auch für seinen Klienten gültig sind. Die Regel, dass keine Kredite vergeben werden, setzt der Verwaltungsarbeit einen weiteren Rahmen.

Über regelmässige Besprechungstermine wird eine Situation geschaffen, in der einem Mangel an finanziellen Mitteln ein Überschuss an Besprechungszeit gegenüber gestellt wird, welcher nicht allein durch den äusseren Ablauf der Geldverwaltung gefüllt werden kann. So entsteht eine Leerstelle, die einen zusätzlichen Raum für die Begegnung mit dem Klienten eröffnet.

Als sich Herr F. an einen ärztlichen Psychoanalytiker wandte, hätte seine Situation kaum verzweifelter sein können. Eine schwere Kokainsucht (Freebase), ein Morbus Parkinson, dessen Behandlung wegen der Sucht nicht möglich war, Schulden in der Höhe von weit über Fr. 300 000. Seine Familie hatte sich von ihm abgewandt und seine Mutter hatte ihn angezeigt, weil er das ihm im Rahmen einer 
Beistandschaft anvertraute Vermögen eines verwaisten, minderjährigen Neffen hinterzogen hatte. Wegen der Krankheit war sein Arbeitspensum auf 50\% reduziert worden, wegen der Sucht war er aber gar nicht mehr arbeitsfähig. Dank guter Anstellungsbedingungen bezog er ein recht hohes Gehalt, das aber gepfändet war. Ein Kollege überwies mir Herr F. zur Durchführung einer Geldverwaltung; er hatte aus einer anderen Zusammenarbeit von unserer Vorgehensweise gehört.

Herr F. ist der Sohn eines bürgerlichen Berufspolitikers, der vor 15 Jahren verstorben ist, und einer Hausfrau, welche sich der Dominanz des Ehemannes unterzog. Er hatte eine vier Jahre ältere Schwester, welche an Krebs verstarb und eine Tochter hinterliess, die durch ihren Tod zurVollwaisen geworden war. Ausserdem hat er eine um drei Jahre jüngere Schwester, welche als Lehrerin in der Nähe der Mutter lebt. Mit ihr hatte Herr F. jedoch praktisch keinen Kontakt. Nach dem Gymnasium enttäuschte er seinen Vater, der von ihm ein Jurastudium erwartet hatte. Statt dessen schrieb er sich in Publizistik ein, beteiligte sich in vorderster Front in der Studentenbewegung der frühen Siebzigerjahre und erntete dafür die Verachtung seines Vaters, um dessen Beachtung und Zuneigung er schon als Kind vergebens gekämpft hatte. Nach dem Studium arbeitete er als Journalist und fand nach seiner Heirat eine Anstellung beim Radio, wo er eine renommierte Informationssendung mit aufbaute. Mit Aufsehen erregenden Recherchen machte er sich einen Namen. Im Rahmen von Reorganisationen wurde sein Arbeitsplatz nach Zürich verlegt, wo er sich eine Wohnung mietete. Die Wochenenden verbrachte er mit seiner Frau und seinen zwei Töchtern in der Nähe seiner Herkunftsstadt. So sei es zu einer gewissen Entfremdung in der Ehe gekommen. Mit Kokain sei er zum ersten Mal in Journalistenkreisen in Kontakt gekommen; zu einer Sucht allerdings sei es allerdings erst viel später, nach Ausbruch seiner Parkinson-Krankheit gekommen und zwar mit Freebase (einer rauchbaren Form von Kokain, auch Crack genannt).

In den ersten zwei Jahren sah ich Herrn F. zweimal wöchentlich für je 50 Minuten; danach ein Jahr lang weiterhin zweimal in der Woche, wobei ein Termin 50 Minuten dauerte, der zweite 10 Minuten. Danach konsultierte er mich etwa zwei Jahre lang wöchentlich, zuerst 50 Minuten, später jedes zweite Mal nur 10 Minuten. Schliesslich reduzierten wir unsere Treffen auf ein monatlich stattfindendes Gespräch.

Es gelang ziemlich schnell, die Lohnpfändung aufzuheben. Einen grossen Teil der Schulden konnte Herr F. über einen Erbvorbezug tilgen; seine Mutter machte ihre Hilfe jedoch von der Geldverwaltung abhängig. Eine Anmeldung bei der Invalidenversicherung wurde von neurologischen und psychiatrischen Gutachten gestützt. Herr F. arbeitete motiviert mit. Sein anfängliches Lebensgeld von Fr. 22.- pro Tag, das ich ihm zweimal wöchentlich bar auszahlte, eröffnete ihm 
kaum Möglichkeiten, sich Kokain zu beschaffen. Nach einem Anfang, in dem er die Geldverwaltung als eine grosse Entlastung empfand und die zur Verfügung stehende Zeit weitgehend mit «technischen» Inhalten gefüllt war, kam es vermehrt zu Klagen über die ungenügenden Mittel und zu Versuchen, mit verschiedenen Begründungen zu Vorschüssen oder zusätzlichen Mitteln zu kommen.

In praktisch jeder Geldverwaltung können wir feststellen, wie nach einer mehr oder weniger kurzen Phase des «Honeymoon» die zuvor getroffenen Abkommen von den KlientInnen schleichend «umdefiniert» werden: Aus derVereinbarung zur Zusammenarbeit nach zuvor bestimmten Regeln wird - für die KlientInnen oft kaum merklich - ein Versprechen derVersorgung gemacht. Damit wird die aufgabenorientierte Arbeit aufgegeben, welche sich an den Bedingungen der ökonomischen Realität orientiert, und es entsteht eine andere, durchaus erwünschte Dynamik. In dieser wird für den Klienten aus dem psychoanalytischen Sozialarbeiter eine omnipotente Figur, welche den Beschränkungen der Realität kaum unterliegt, sich aber weigert, dem immer stärker werdenden Wunsch nach einer möglichst kompletten Versorgung nachzukommen. Immer mehr erscheint es dann den KlientInnen, als sei an den knappen materiellen Verhältnissen die Geldverwaltung und damit der psychoanalytische Sozialarbeiter schuld. So entstehen nicht selten heftige Gefühle von Wut auf den Sozialarbeiter, die bei ihm wiederum Ärger und Schuldgefühle auslösen können. Diese können dann schnell als Vorwürfe wieder an den Klienten zurück gegeben werden, was die Dynamik noch verschärft.

Auch Herr F. versuchte in dieser Phase der Arbeit unter Verleugnung der Bedingungen der äusseren Realität Geld zu beziehen, das nicht vorhanden war. So wiederholte sich im Rahmen der Geldverwaltung das, was zur Geldverwaltung geführt hatte: Die Herrschaft der Jetzt-Zeit mit ihren imperativen Wünsche und der sie begleitenden imperativen Affekte, ganz wie in der Gier der Kokainsucht.

In den Worten Winnicotts befand sich Herr F. in einem Zustand, in dem er nicht spielen kann. Auch in unserem Konzept von Geldverwaltung gilt, "dass die Arbeit des Therapeuten dort, wo Spiel nicht möglich ist, darauf ausgerichtet ist, den Patienten aus einem Zustand, in dem er nicht spielen kann, in einen Zustand zu bringen, in dem er zu spielen imstande ist.» (Winnicott 1987: 49). Damit der Raum des zeitlichen Überschusses vom Klienten als potentieller Raum im Sinne Winnicotts genützt werden kann, müssen vor allem zwei Bedingungen erfüllt sein: Die Sicherheit, dass seine imperativen Affekte vom psychoanalytischen Sozialarbeiter ausgehalten und «verdaut» werden können und dass eine Antwort möglich ist, die insofern angemessen ist, als sie die innere Not anerkennt und gleichzeitig zum Schutz des Patienten die äussere Realität angemessen berück- 
sichtigt. Wenn dies gelingt, kann der Klient mit der Zeit die äussere Realität durch eine Differenzierung seiner inneren Verfassung differenzierter wahrnehmen, indem sie ihm Spielräume preisgibt, die er zuvor nicht erkennen konnte oder denen er kaum Bedeutung zumessen konnte.

Es ist also die Aufgabe des psychoanalytischen Sozialarbeiters, die Gefühle des Klienten und die dadurch hervorgerufenen Gefühle in sich selber auszuhalten, zu «verdauen» und sie in verträglicher Form wieder in die Arbeit einzuspeisen. Ich versuchte dies mit Interventionen wie dieser: «Wir hatten zu Beginn der Arbeit ja ganz genau besprochen, welches unsere Aufgaben und Möglichkeiten sind. Das hat ja auch ganz gut geklappt und ich habe auch den Eindruck, dass Sie meist froh sind, dass mit der Geldverwaltung an die Stelle der katastrophalen Not, welche unsere Arbeit notwendig gemacht hat, die Enge einer neuen Normalität getreten ist, in welcher gewiss noch viele Wünsche lange zuwarten, andere vielleicht auch gänzlich unerfüllt bleiben müssen. Ich sehe ja, dass Sie trotz all dieser Schwierigkeiten immer wieder gut mit mir zusammenarbeiten und ich schätze das auch sehr. Und gerade deshalb frage ich mich immer wieder, wie es wohl dazu kommt, dass unsere Vereinbarung immer wieder sozusagen in sich zusammenfällt, Sie die Geldverwaltung trotz Ihres zweifellos meistens vorhandenen Willens zur Mitarbeit manchmal nur noch schwer als Hilfe wahrnehmen können und dann - so scheint mir - Sie Ihre Enttäuschung in einerWeise zum Ausdruck bringen müssen, welche nahe legt, dass Ihre Not mit der Geldverwaltung begonnen hätte, obwohl wir ja beide wissen, dass dem nicht so ist.» Mittels derartiger Interventionen, welche in abgewandelter Form oft wiederholt werden mussten, gelang es immer wieder, die Situation zu entspannen und die Mitarbeit der Klientinnen wieder zu gewinnen. Insbesondere hatte das Gefühl, verstanden zu werden, zur Folge, dass die Wut nachlässt und einer oft traurig gestimmten Nachdenklichkeit weicht. Die auf diese Weise wieder erlangte Fähigkeit zu spielen, kann sich in glücklichen Fällen dann darin ausdrücken, dass Ideen auftauchen, wie scheinbar unerfüllbare Wünsche mit den zur Verfügung stehenden Mitteln in anderer Form teilweise doch befriedigt werden können. So hat sich Herr F. im Laufe der Gespräche an seine Liebe zur Querflöte erinnert, die er aber in seiner früheren Geldnot versetzt hatte. Mit Gesuchen an Stiftungen wurde dann der Kauf einer neuen Flöte möglich. Beim Musizieren fühlte er sich - wie vordem nach der Einnahme von Kokain - von seiner Parkinson-Krankheit weitgehend unbehelligt.

Die Rückfälle wurden zwar seltener, aber sie hielten über lange Zeit an und führten immer wieder dazu, dass Herr F. manchmal auch ausserhalb der vereinbarten Termine ziemlich ultimativ Geld verlangte. Mit der Zeit konnte er mir mitteilen, 
dass er den Stoff nicht wie allgemein üblich «auf der Gasse» erstand, sondern dass der Kokainkonsum an Besuche in einer Art von Bordell geknüpft war. Es handelte sich um ein Etablissement, in dem «umgebaute» Brasil-Prostituierte, die selber schwer kokain- und spielsüchtig waren, ihren Kunden Kokain in rauchbarer Form verkauften. Zu eigentlichen sexuellen Handlungen kam es nie, aber Herr F. hatte sich dort vor dem Beginn der Geldverwaltung immer mehr verschuldet, indem er in dieser sexuell aufgeladenen Stimmung in einer Nacht für mehrere Tausend Franken Kokain für sich und seine Angebetete ausgab. Die Suchterkrankung seiner Geliebten hatte zur Folge, dass diese immer wieder vor Gläubigern fliehen musste und dann manchmal - auch später, als Herr F. kein Kokain mehr konsumierte - für Tage oder garWochen bei Herr F. lebte, ohne dass es dabei zu sexuellen Handlungen gekommen wäre. Die transsexuellen Prostituierten seien aufgrund ihrer Ausgrenzung durch ihre Landsleute, die Illegalität ihrer Geschäfte und ihre mehrfache Abhängigkeit (Kokain-, Medikamenten- und Spielsucht) besonders verletzlich und werden deshalb besonders brutal ausgebeutet.

Mit diesen Begebenheiten lieferte mir Herr F. nicht nur neues Material zum Verständnis seiner damaligen Situation und zu den unbewussten Hintergründen für sein Verhalten, er zeigte mir gleichzeitig auch, dass er unser Setting verstanden hatte und sich mit ihm identifizieren konnte. Die Parallele zwischen seiner Geschichte aus dem Bordell und der Situation unserer Geldverwaltung war nämlich unübersehbar. Hier wie dort wurde zu einem konkreten Zweck ein Setting geschaffen, in dem es dann aber um anderes ging. Hier wie dort kam es zu einer Verlagerung, zu einer Verschiebung, zu einer Übersetzung und zu einer Übertragung von einer Situation zu einer anderen und immer noch weiteren, von einem Wunsch zu anderen und immer noch mehr, hier wie dort ergab sich aus der Kette der Verschiebungen und Übersetzungen ein immer weiter gehendes Verständnis der Situation von Herrn F. Aus der Geldverwaltung wurde ein Setting, in dem er immer mehr von sich erzählen konnte, so dass immer deutlicher wurde, wie seine jetzige Not mit der Situation in dem Bordell, wie diese wieder mit seiner Familiensituation und diese mit unbewussten, inzestuösen Konflikten und ihrem Schicksal miteinander verknüpft waren.

Darüber hinaus wurde auch deutlich, wie sich die libidinösen Wünsche und Konflikte in immer neue Formen wandeln und giessen und dabei natürlich auch immer einer Ausbeutung - wenn man so will - ausgesetzt sind. Auch das nicht nur im Bordell, sondern auch in unserer Situation, in der ja die Geldverwaltung auch dazu gebraucht - oder missbraucht? - wurde, um in eine analytische Situation mit Herrn F. zu gelangen. 
Diese Information verband sich für mich mit Mitteilungen, die er mir bei anderen Gelegenheiten gemacht hatte: Häufig hatte sein Vater vor den Kindern aber auch vor aussen stehenden Dritten die Mutter in grober Weise herabgesetzt und gedemütigt, was den Sohn mit ohnmächtiger Wut erfüllt hatte. Darüber hinaus hatte der Vater, von dem sich Herr F. stets abgelehnt gefühlt hatte, seine ältere Schwester über mehrere Jahre hinweg (vom 14. bis zum 18. Lebensjahr) sexuell missbraucht. Herr F. hatte davon Kenntnis und war einmal sogar Zeuge davon. Die Schwester hatte der Mutter davon berichtet, doch die Mutter wollte davon nichts wissen. Später ging sie eine unglückliche Ehe mit einem Mann ein, der an einem Unfall verstarb, als ihre gemeinsame Tochter fünf Jahre alt war. Weitere zehn Jahre später verstarb auch sie an Krebs. Für Herrn F. war klar, dass ihre Erkrankung und ihr früher Tod Folge des Missbrauches waren. Er übernahm die Verwaltung des nicht unbeträchtlichen Vermögens des Neffen und veruntreute später davon eine grosse Summe wegen seines Kokainkonsums in besagtem Bordell, was dann zur Anzeige von Herr F. durch seine Mutter und zu seinerVerurteilung führte, in deren Zusammenhang meine Arbeit mit Herr F. für zwei Jahre als ambulante Massnahme deklariert wurde.

In seiner erfolgreichen Zeit als Radio-Journalist war die Aufdeckung von Skandalen eine Leidenschaft von Herr F, für die er sehr geschätzt wurde, die es ihm aber auch schwer machte, die Arbeit im «normalen» News-Bereich zu besetzen; er empfand diese Normalität als lästige Routine. Der Kokainkonsum von Herr F. bescherte ihm für Minuten die Vorstellung, von seiner Parkinsonkrankheit geheilt zu sein, weil er für kurze Zeit keinerlei Blockaden verspürte und das Gefühl, sich absolut frei bewegen zu können. Nach den Konsum-Exzessen gesellten sich zu den allgemein bekannten Entzugserscheinungen dann aber verstärkte Blockaden.

Der Kokaingebrauch von Herr F. war also überdeterminiert: Für kurze Zeit konnte die Illusion von körperlicher Gesundheit geträumt werden, gleichzeitig war die Strafe durch die Verschlimmerung der Blockaden schon vorbereitet. Ein anderer Grund war die spezielle Situation in besagtem Bordell. Als «Männer in nachgebildeten Frauenkörpern» stehen die jungen Prostituierten, mit denen er Kokain konsumierte, nämlich im Zentrum des Begehrens von - in der Regel - älteren Männern. Einerseits begehrte Herr F. diese so, wie sein Vater seine Schwester (statt ihn selbst) begehrt hatte, andererseits blieb die inzestuöse Dimension des Erlebens eingegrenzt, indem an die Stelle der sexuellen Handlungen der gemeinsame Kokain-Konsum trat. Die Illegalität des gemeinsamen Kokain-Konsums verwies aber immer auf das Verbotene des inzestuösen Begehrens. Indem er die verfolgten Prostituierten bei sich aufnahm, konnte er ihnen aber auch den Schutz bieten, den 
seine Mutter der missbrauchten Schwester nicht geben konnte. Auch seine Arbeit bekam in diesem Zusammenhang eine zusätzliche Bedeutung: Als aufdeckender Journalist lieferte Herr F. nämlich manchen Würdenträger der öffentlichen Kritik aus und lüftete damit in gewisser Weise immer wieder das Familiengeheimnis, um die Mauer des Schweigens zu durchbrechen.

Es stellte sich nun die Frage, wie dieses Material im Rahmen einer Geldverwaltung aufgegriffen werden könnte. Was konnte man tun, um das Verhältnis von gemeiner Lebenswirklichkeit und inneren Fantasien besser zu verknüpfen?

Ich entschied mich dafür, am prekären Verhältnis von Herrn F. zu seiner Mutter anzusetzen. Er hatte mir erzählt, dass er sie in jüngster Zeit mehrfach auf den sexuellen Missbrauch der älteren Schwester angesprochen habe; sie sei wortlos vom Essen aufgestanden und in ihr Zimmer gegangen, das sie dann erst nach seinem Weggang wieder verliess. Sie war überdies mit der Aufgabe der Geldverwaltung unmittelbar verbunden, indem sie über einen Erbvorbezug die Tilgung eines grossen Teils der Schulden ermöglichte, insbesondere jener, die durch die Veruntreuung des Vermögens des Neffen von Herrn F. entstanden waren. In der Geldverwaltung erschien der Erbvorbezug als ein verzinsliches Darlehen und ich überwies ihr monatlich Zinsen von fast tausend Franken. Der mündliche Darlehensvertrag zwischen Mutter und Sohn ging von Zinsen in der Höhe eines Anlagekontos aus. Inzwischen waren jedoch die Zinssätze stark gefallen und es stellte sich die Frage einer Anpassung. In vorabklärenden Telefongesprächen hatte ich den Eindruck gewonnen, dass die Mutter die Parkinson-Erkrankung ihres Sohnes kaum zur Kenntnis genommen hatte, nur die Kokainsucht als Ursache für seine Invalidisierung gelten liess und der dramatische Rückgang des Kokainkonsums keine Beachtung fand. Alle Verbesserungen, die ja unübersehbar waren, schrieb sie allein meiner «klaren Führung» des Sohnes zu. Auch von ihrer Seite her waren also Vorstellungen von Schuld im Spiel (ihre Schuldgefühle gegenüber der verstorbenen Tochter, die im Gespräch mit dem Sohn zur wortlosen Flucht geführt hatten), welche fast jedeWahrnehmung der inzwischen doch deutlich veränderten Umstände der Realität des Lebens ihres Sohnes hartnäckig verhinderten.

Wir luden die Mutter zu einer Besprechung in meiner Praxis ein, setzten einen Vertrag mit variablen Zinskonditionen auf und ich erzählte ihr von unserer Arbeit und vom grossen Erfolg, der ohne ihre Hilfe und ohne die engagierte Mitarbeit ihres Sohnes absolut undenkbar gewesen wäre. Ich fügte an, dass ich von Herr F. wisse, dass es Themen gäbe, die zwischen ihnen nicht besprechbar seien und das würde mich eigentlich nichts angehen. Aber es sei mir ein Anliegen, ihr mitzuteilen, dass Herr F. - auch wenn ihm dieses Verschweigen noch immer schwer 
falle - mir gegenüber immer klar gesagt habe, dass er sich sicher sei, dass sie, seine Mutter, mit dem, was sie in und um sich vorgefunden habe, stets das Bestmögliche zu machen versucht habe. Es sei mir nicht möglich, ihnen hier einen kleinen Imbiss anzubieten und mir fehle leider dazu auch die Zeit, doch ich sei überzeugt, dass Herr F. und sie vor der Abreise noch Zeit für ein Gespräch in einem ruhigen Café hätten, was sie dann auch taten, beide mit Tränen in den Augen.

Dieses kurze Begegnung trug zum Abbau der Spannungen und Vorwürfe bei. Herr F. konnte sich inzwischen ein Generalabonnement bei der SBB leisten und besuchte von da an seine Mutter regelmässig. Als sie schwer erkrankte, blieb er sogar über mehrere Tage in ihrer Wohnung und sorgte für sie, was einen Aufenthalt in einer Reha-Klinik überflüssig machte. Über den Missbrauch haben sie bis zum Abschluss meiner Arbeit mit Herr F. nicht gesprochen, das Schweigen aber war von einem Verschweigen zu einem unausgesprochenen Eingeständnis der Realität des Vergangenen geworden. Bei meinem letzten Kontakt mit der Mutter von Herr F. ich hatte wegen eines Versehens die monatliche Zinszahlung doppelt ausgeführt - sagte sie mir, Herr F. sei «so erwachsen geworden». Auf diese Weise brachte sie zum Ausdruck, dass es Herrn F. möglich geworden war, seine Mutter nicht mehr nur fast ausschliesslich mit dem Verrat an der missbrauchten Schwester und an ihm selber gegenüber der Polizei (im Zusammenhang mit der Hinterziehung vom Vermögen des Neffen) in Verbindung zu bringen. Zwar ärgerte er sich in dieser Zeit über ihre Weigerung, die Dienste einer Pflegerin in Anspruch zu nehmen, doch als das Fortschreiten seiner Krankheit gewisse Hilfeleistungen zur Zumutung werden liessen, konnte sie sich dann schliesslich doch dazu entscheiden, eine Pflegerin zuzulassen. Zu seiner Entlastung, wie sie sich ausdrückte. Sie machte von sich aus das Angebot, dass sie über eine Aufstockung des Darlehens die dringend notwendige Gebiss-Sanierung vorfinanzieren würde, was die äusseren Male der einstigen Verwahrlosung zum Verschwinden brachte und zur Folge hatte, dass Herr F. sicherer auftreten konnte und vom Stigma der Drogenabhängigkeit auch in dieser Beziehung befreit war.

Es war bei diesem (einzigen) Treffen zusammen mit der Mutter gelungen, eine gerechtere Lösung der anstehenden Probleme innerhalb des engeren Aufgabenbereiches der Geldverwaltung zu finden (Höhe der Verzinsung des Darlehens). Darüber hinaus aber war es vor allem gelungen, über die Andeutung des Themas vom Missbrauch der älteren Schwester durch den Vater und die gemeinsame Trauer darüber, «das Verhältnis von gemeiner Lebenswirklichkeit und inneren Fantasien» neu zu verknüpfen, was sich meines Erachtens in den Veränderungen der Beziehung von Herr F. zu seiner Mutter deutlich ausdrückte. 
Auch die Tatsache, dass Herr F, der bisher in unmittelbarer Nähe von besagtem Bordell (und zur Drogenszene) gewohnt hatte, in dieser Zeit eine neue Wohnung suchte und in einem «bürgerlichen» Wohnquartier fand, interpretiere ich als Anzeichen für «eine bessere Verknüpfung dieser beiden Wirklichkeitsbereiche» ("gemeiner Lebenswirklichkeit und inneren Fantasien»). Ebenso kam es zu einer Entkrampfung der Beziehung zu seiner getrennten Ehefrau und zur Belebung seiner Beziehung zu den beiden erwachsenen Töchtern. Auch das Wiederanknüpfen von Beziehungen zu früheren ArbeitskollegInnen und zu alten Bekannten sehe ich in diesem Zusammenhang.

Nach etwa vier Jahren hatte sich die Situation stark verändert: Dank seiner guten sozialen Absicherung (Invalidenrente, Hilflosen-Entschädigung, BVGRente) und durch die Reduktion seiner Schulden hatte sich sein verfügbares Lebensgeld verdreifacht, die nun sehr selten gewordenen Rückfälle blieben in ihren Auswirkungen begrenzt, weil die Auszahlung dieses Geldes bis anhin in zwei Raten pro Woche aufgeteilt wurde. Herr F. stellte in der Zwischenzeit ein monatliches Budget auf, in dem alle regelmässigen und einmaligen Zahlungen sowie die notwendigen Rückstellungen berücksichtigt waren und die Höhe des Lebensgeldes bestimmt wurde, das nun nicht mehr bar, sondern zunächst noch in zwei Raten pro Woche, bald aber in einer Wochenrate auf ein nicht überziehbares Konto überwiesen wurde. Die ambulante Massnahme, die im Zusammenhang mit seiner Verurteilung wegen der Hinterziehung des Vermögens seines Neffen ausgesprochen worden war, konnte aufgehoben werden und es stellte sich das Thema der Beendigung der Geldverwaltung. Nach weiteren zwei Jahren sandte mir Herr F. die Aufstellung seiner Rechnungen und die Höhe sowie den Stichtag der zu überweisenden Lebensgeld-Raten. Einzig seine Einnahmen liefen noch über das Konto unseres Vereines und die Rückstellungen und die Verwaltung der Krankenkassen-Angelegenheiten blieben in meiner Verwaltung. Wir sahen uns inzwischen noch monatlich einmal zu einem Gespräch, wo die Beendigung der Geldverwaltung das Thema war.

Die Arbeit mit Herr F. war gegenüber seiner Krankenkasse als delegierte Psychotherapie deklariert, wobei die Geldverwaltung ausdrücklich Erwähnung fand und die administrative Hintergrund-Arbeit zu einem gewissen Teil als «Leistung in Abwesenheit des Patienten durch den delegiert arbeitenden Psychotherapeuten» in Rechnung gestellt wurde. Das war Herr F. natürlich bekannt. Ich legte ihm jeden Monat eine Kopie meiner Rechnung an die Krankenkasse vor, ausserdem bestand jederzeit Einsichtsrecht in die gesamten Unterlagen der Geldverwaltung. 
Als er ganz am Ende unserer Zusammenarbeit dann die Verwaltung seiner Krankenkassen-Abrechnungen wieder selber übernommen hatte, bat er, eine Woche vor dem vereinbarten Ende der Geldverwaltung, dringend um einen Termin. Er war in grosser Erregung und brachte vor, dass er die gesamten Rechnungen, die ich seit Beginn der Geldverwaltung der Krankenkasse gestellt habe, gesichtet hätte und dass er einem grossen Betrug meinerseits auf die Schliche gekommen sei, weil die verrechneten Leistungen bei weitem die erbrachten übersteigen würden. Er selber sei davon ja nicht direkt betroffen, aber es sei seine Aufgabe, die Interessen der Krankenversicherung wahrzunehmen und er müsse mich deswegen wohl anzeigen. Als möglichen Ausweg würde er es akzeptieren, wenn ich eine Zahnarzt-Rechnung in der Höhe von Fr. 2500._- die durch das Darlehen der Mutter nicht gedeckt war, übernehmen würde, so dass wenigstens ein Teil der durch mich ertrogenen Mittel der Krankenversicherung zu seinen Gunsten Verwendung finden würden. Ich war perplex und zutiefst erschrocken, fragte mich sofort, ob ich vielleicht etwas übersehen hätte. Dann wurde ich wütend und enttäuscht über die meines Erachtens völlig ungerechtfertigten Unterstellungen. Mir ging in kurzer Zeit durch den Kopf, wie viele Stunden (vor allem zu Beginn der Arbeit) von mir nicht hatten verrechnet werden können und wie ich bis zum Zeitpunkt, wo Herr F. wieder einigermassen gut leben konnte, mit seinem Wissen auf die Verrechnung von Franchise und Selbstbehalten verzichtet hatte. Es gelang mir gerade noch, äussere Ruhe zu bewahren und in sachlichem Ton meinen Standpunkt vorzubringen. Ich bot ihm an, den fehlenden Betrag für die Gebiss-Sanierung mit Gesuchen an eine gemeinnützige Stiftung zu beschaffen, was Herr F. als Eingeständnis meiner Schuld wertete. Er werde aber von einer Anklage absehen, waren seine letzen Worte, bevor er sich doch noch mit einem knappen Dank für die Hilfe verabschiedete.

Das war natürlich ein Schock, der nicht einfach zu nehmen war. Es war ja klar, dass seine Vorwürfe von der Sache her nicht haltbar waren, aber sie wiesen auch darauf hin, dass etwas in der Tat noch offen war. Sie wiesen auch darauf hin, dass die Versöhnung mit seiner Schuld, mit seinen Konflikten und mit seiner Gier, mit seiner Sucht doch nicht ganz vollzogen war. Es war doch noch nötig für ihn, mich zum Betrüger zu machen, mich zu entlarven, so wie er es als Journalist, mit prominenten Figuren gemacht hatte, weil er seine eigene Schuld nicht ganz ertragen konnte. Da blieb ein Rest, der nicht aufgelöst werden konnte, so wie immer ein Rest bleibt, der nicht aufgelöst werden kann, so wie der Vorwurf der Ausbeutung eben zur Sexualität und zur Triebhaftigkeit gehört und nicht einfach in Wohlgefallen aufgelöst werden kann. Damit müssen wir leben. Er und ich. 
Erst in der nachträglichen Betrachtung ist mir allmählich klarer geworden, dass ich in der letzten Phase unserer Arbeit zwar die technischen Aspekte der Beendigung sorgfältig geplant und besprochen hatte, die Bedeutung der Trennung für Herr B. jedoch unterschätzt hatte. Die Arbeit der Geldverwaltung war ja gewiss eine Art Rettung vor dem finanziellen und gesundheitlichen Ruin für ihn gewesen. Es war Herr F. aber nicht möglich geworden, eine neue Liebesbeziehung einzugehen und das Fortschreiten der Krankheit trieb in oft in Verzweiflung und ohnmächtige Wut, wenn er trotz der guten medizinischen Betreuung nun immer stärker von Blockaden heimgesucht wurde; er erlebte die Weigerung seines Körpers, seinem Willen zu gehorchen, als tiefe Kränkung und zunehmende Bedrohung und er beschäftigte sich seit einiger Zeit, auch in Gesprächen mit einem ehemaligen Berufskollegen, der an derselben Krankheit in einem weit fortgeschritteneren Stadium litt, über den «richtigen» Zeitpunkt, seinem Leben ein Ende zu setzen.

Als sich Herr F. vor x Jahren in grösster psychosozialer Not mit der Absicht, eine Psychoanalyse zu machen, um seiner Probleme Herr zu werden, an meinen ärztlichen Kollegen wandte, war er wohl auch von unrealistischen Heilungserwartungen getrieben. Die Mängel meiner Arbeit in der Abschlussphase der Geldverwaltung, die in erster Linie darin bestanden, dass die Trauer über den «unheilbar gebliebenen Rest» in Bezug auf sein Liebesleben, vor allem aber auch in Bezug auf die Krankheit nicht genügend durchgearbeitet werden konnte, was dazu führte, dass ich die Trennung vor allem als ein «technisches Problem» behandelte, liessen Herr F. nur die Möglichkeit, die doch weitgehend gelungene Arbeit am Ende als «Betrug» zu entwerten. Die Frage, wo die Grenzen einer Geldverwaltung im Rahmen von psychoanalytischer Sozialarbeit liegen, muss deshalb in diesem Punkt offen bleiben.

\section{Literatur}

Leuthard, Esther, Bader, Heinrich (2005): Trojanisches Pferd. In: Verein für Psychoanalytische Sozialarbeit (Hrsg.), Entwicklungslinien psychoanalytischer Sozialarbeit, Tübingen: Edition Diskord. 132-149.

Michael, Günter (2005): Leicht beieinander wohnen die Gedanken, doch hart im Raume stossen sich die Sachen. In: Verein für Psychoanalytische Sozialarbeit (Hrsg.), Entwicklungslinien psychoanalytischer Sozialarbeit, Tübingen: Edition Diskord. 84-100.

Winnicott, Donald Woods (1987): Spielen eine theoretische Darstellung. In: Vom Spiel zur Kreativität, Stuttgart: Klett-Cotta. 49-64. 